\title{
DEVELOPMENT OF INTELLIGENT EQUIPMENTS FOR PRECISION AGRICULTURE
}

\author{
Xiaochao Zhang ${ }^{*}$, Xiaoan Hu, Wenhua Mao \\ Institute of Machine and Electron Technology, Chinese Academy of Agricultural Mechanization \\ Sciences, Beijing, China, 100083 \\ * Corresponding author, Address: P.O. Box 25, Institute of Machine and Electron \\ Technology, Chinese Academy of Agricultural Mechanization Sciences, No. 1, Bei Shatan, \\ Deshengmen wai, Beijing, 100083, P. R. China, Tel: +86-10-64882584, Fax: +86-10-64882652, \\ Email: zxc@caams.org.cn
}

\begin{abstract}
This paper briefly described the general production of intelligent agricultural machine in precision agriculture. It summarized the basic principle and the application in precision agricultural demonstration field of those agricultural machines, which mainly included wheat variable ferti-seeder, yield distribution information acquiring system, variable controlled large irrigation system moving in synchronous, intelligent spraying herbicide machine, ultralow altitude remote system, fast-analysis system of food quality and on-board computer, GPS, GIS specially designed for precision agriculture.
\end{abstract}

Keywords: precision agriculture, variable ferti-seeder, yield distribution, variable controlled, intelligent spraying

\section{INTRODUCTION}

Precision agriculture means using the soil character of every field and the growth character of some one crop to decide the investment of agricultural resources, such as the most seeds, fertilizer and irrigation, and than acquiring the maximum benefit both in economics and environments. The key character of precision agriculture lies in correct prescription, veracious position and accurate agricultural resource inputs.

Intelligent agricultural machine plays a very important role in the research of precision agriculture technology. According to the conception of systematization, intelligent agricultural machine is composed of agricultural machine

Zhang, X., Hu, X. and Mao, W., 2008, in IFIP International Federation for Information Processing, Volume 259; Computer and Computing Technologies in Agriculture, Vol. 2; Daoliang Li; (Boston: Springer), pp. 951-958. 
with electronics, intelligent control and information technology, to accomplish the function that normal agricultural machine cannot do.

\section{ON-BOARD COMPUTER AND NAVIGATION AND POSITION SYSTEM BASED ON GPS FOR PRECISION AGRICULTURE}

Navigation system which was based on the GPS position technology navigates agricultural machine in field according to the scheduled course and navigation indication (Liu, 2003). This system can offer scheduled course for operators of farming plane and tractor, and show the error range of yawing. If the DGPS system was composed of a low-cost OEM border and Pseudo-Range Difference method, the position precision, error range of it could be 2 meters (RMS). If the Carrier Phase Real-time Kinematics Differential Method (RTK) was used, the result of it could be 2 centimeters. The cost of GPS navigation and position will be lower in districts, where have the difference station or can offer network difference data.

In figure 1, a precision agricultural on-board computer together with GPS receiver, which can be used precision agriculture.

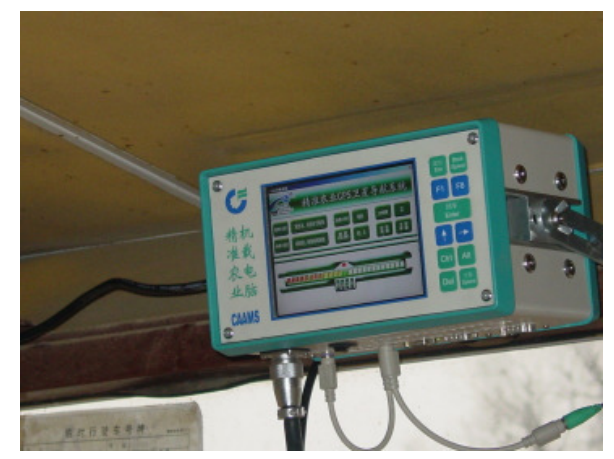

Figure 1. An automatic navigation system based on GPS

\section{AUTOMATIC VARIABLE FERTI-SEEDER}

The equipment which can adjust fertilizer quantity dynamically was developed for the large scale variable ferti-seeder. It can real-time adjust the amount of fertilizer according to the given prescription map and control instruction. That could improve the reliability of dynamic work and the equipment level of information. 


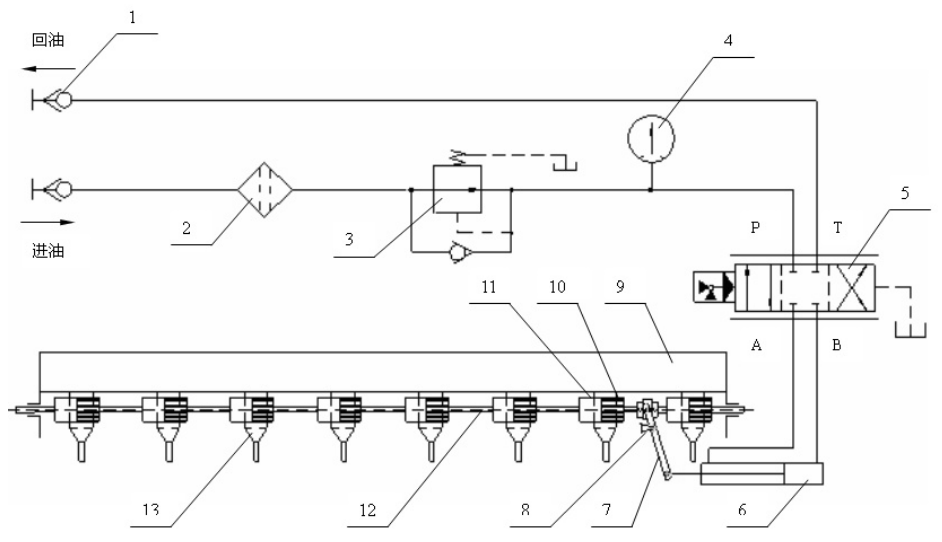

Figure 2. Wheat variable ferti-seeder

1-hydraulic pressure pipeline's tie-in of input and output, 2-filter, 3-one-way relief pressure valve, 4-manometers, 5-electro-hydraulic servo valve, 6-two acting oil cylinder, 7-fertilizer quantity setting lever, 8- pivot, 9-fertilizer trunk, 10-fertilizer ejector wheel, 11-block wheel, 12-fertilizer ejector axis, 13-fertilizer ejector box

In figure 2, the control equipment operated by electro-hydraulic system was geared into the tractor's hydraulic pressure system. When the variable ferti-seeder worked in the field, the control system could real-time adjust the quantity of fertilizer according to the prescription. When the quantity of fertilizer was needed to increase, the electro-hydraulic servo valve pushed the pressure oil from the port A to the left of two acting oil cylinder, then pressed the piston pole to move rightwards. With that, the fertilizer quantity setting lever turned around the pivot counter-clockwise. another end was transversely linked with the fertilizer ejector axis. At the same time, the fertilizer ejector axis moved leftwards that opposite to the fertilizer trunk. The fertilizer ejector wheel moved leftwards, too. Therefore, the amount of fertilizer was increased. If the quantity of fertilizer was needed to decrease, the electro-hydraulic servo valve pushed the pressure oil from the port B to the right of two acting oil cylinder. Then the fertilizer quantity setting lever turned around the pivot clockwise. At last the work length of the fertilizer ejector wheel was decreased. As a result, the fertilizer quantity was reduced. If the quantity of fertilizer was needed to be a constant, the electro-hydraulic servo valve controlled the pressure balance of port A and port B for the piston of two acting oil cylinder could not move. The amount of fertilizer would be invariable, accordingly.

The controls parameters included that the fertilization adjustable range ( $75-375 \mathrm{~kg} / \mathrm{ha})$, precision ( $\pm 5 \% \mathrm{FS})$, fertilization system response interval $(<30 \mathrm{~s})$, seed quantity adjustable range $(90-225 \mathrm{~kg} / \mathrm{ha})$, seed system response interval $(<42 \mathrm{~s})$. 


\section{YIELD DISTRIBUTION INFORMATION ACQUIRING SYSTEM}

An unprecedented method based on weight was invented to get the yield distribution information. The method could increase the veracity of grain flux survey. According to the characteristic of grain transmission in the traditional combine, the yield sensor was composed of helix churn-dasher weight equipment. The dynamic weighing method could insure the precision of measure.

Operation principle: A lifter sent grain to spiral propeller, which drive grain into the case horizontally. The weight of spiral propeller, drive equipment and flowing grain was measured by the weight sensors, after being amplified by a high-accuracy amplifier, transformed to digital format, then sent into on-board computer. After modified by moisture information, the weight information could be turned into yield information by integral calculation. Finally, the yield map would be mapped according to the yield data, together with GPS positioning system. To improve the precision of measurement, signal processing technology was used, including the wavelet signal filtering, multi-dimension vibration signal compensating, GPS signal postprocessing, etc.

Experimentation in figure 3 tell us, weight based yield obtained system achieved an precision of $\pm 3.5 \%$.

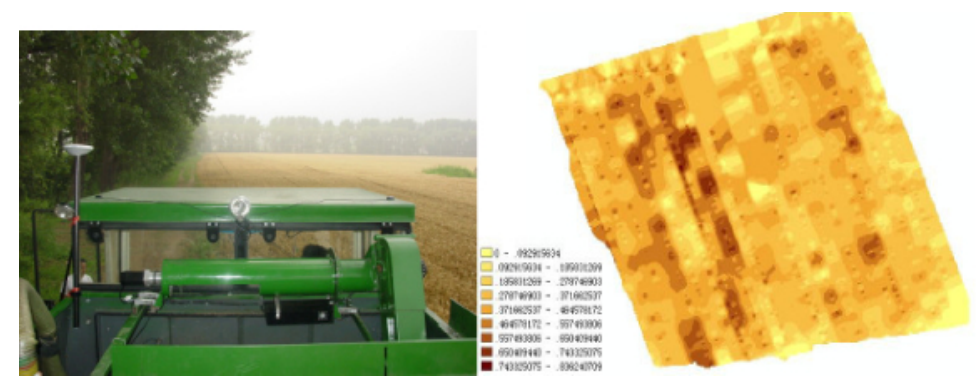

Figure 3. Food yield distributing information acquired system

\section{VARIABLE CONTROLLED SYSTEM FOR LARGE IRRIGATOR IN LINEAR MOVE}

The GPS OEM board based high precision navigation and positioning technology, together with the collection and analysis of soil moisture, prescription map outputted by the Decision Support System, and remote survey and control system of large-scale irrigator, realized the goal of variable 


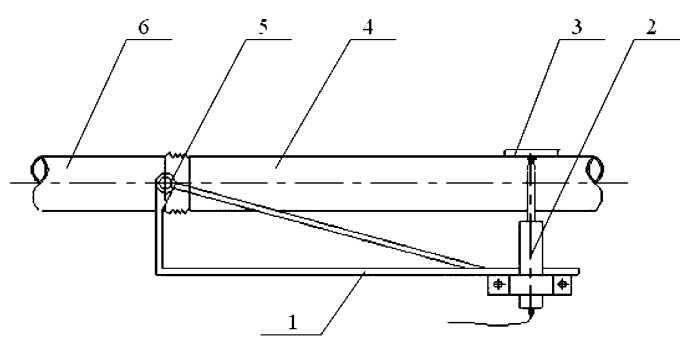

Figure 4. A control construction of large irrigation system

controlled irrigation in precision agriculture. Peasant could control largescale irrigation machine in office to make it automatically work in field without manned. And the irrigation quantity could be automatically adjusted according to the given or anticipant data in the specified area of field.

The method of return-to-zero feedback of every variety angle included two aspects (Zhang et al., 2004). One was angle sensed in synchronous, another was feedback controlled. The method of angle sensed in synchronous was shown in figure 4.

For every section of a large irrigator could move in synchronous, every angle between two sections was measured. If it was not zero, control system of on-board computer would send adjust signals to every frequency conversion governor respectively. That would adjust the speed of ground wheels to return every angle to zero. As the response time is quite short, and every angle is quite small, the angles between every section could be kept under $0.05^{\circ}$.

The variable control of large-scale irrigation system also included: remote sensing and remote control of irrigation machine, rotate speed of every ground wheel of each section adjusted forward or backward to change the stance of irrigation machine, digitized accurate control technology used to control irrigation quantity in the field according to the given prescription map, automatic navigation control and fuzzy decouple control of every speed of rotating ground wheel, etc.

\section{INTELLIGENT SPRAYING MECHANISM BASED ON AUTOMATIC WEED DETECTION}

An automatic detection method of weed image, a control method of integration of mechanics and electrics, and a weeding equipment of intelligent and precision spraying herbicide were developed. When the spraying mechanism was worked in the field, it could real-time open the relevant nozzles and variably quantity of herbicide according to the species and distribution of 


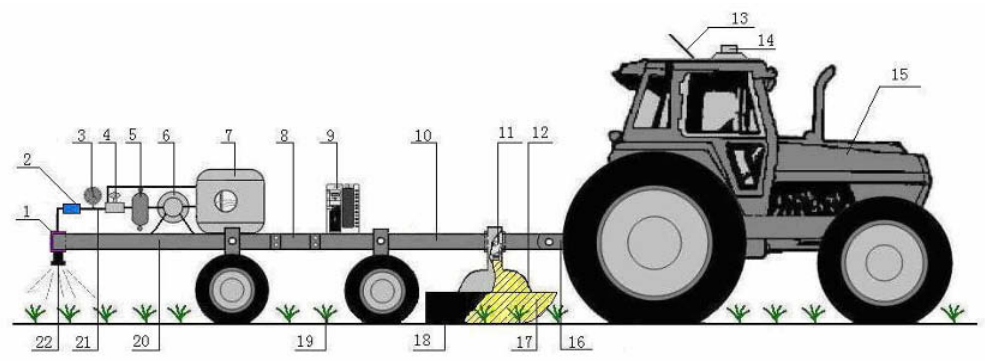

Figure 5. An intelligent weed detection and spraying herbicide machine 1-electromagnetic valves, 2-flow control valves, 3-pressure meter, 4-pressure regulator valve, 5-filter, 6-liquid pump, 7-herbicide trunk, 8-linking frame, 9-computer, 10-frame of image capturing, 11-cameras, 12-close box, 13-antenna of DGPS, 14-GPS, 15-tractor, 16-traction frame, 17-lamp lighting, 18-rubber shield, 19-green plant, 20-spraying frame, 21-pipeline, 22-nozzles

weed infestation. The aim of it was saving herbicide and reducing environmental pollution. The spraying system based on automatic detection of weed images was composed of image detection equipment and spraying equipment (Figure 5).

The image detection equipment was included the frame of image capturing, cameras, box closed in, lamp lighting, rubber shield, and so on. The multi-cameras were transverse mounted on the frame of image capturing. The spraying equipment was made up of electromagnetic valves, flow control valves, pressure meter, pressure regulator valve, filter, liquid pump, herbicide trunk, spraying frame, pipeline and nozzles, etc. The nozzles were corresponded to the electromagnetic valves. A pairs of them were a suite connecting with a flow control valve. The suites were matching with the breadth of captured images. The whole machine was formed by the image detection and the spraying equipment which was linked with the traction frame. The computer processed the control of weed detection and spraying automatically.

The method of automatic detection of crop and weed was done as follow steps:

1) Segmentation of green plant and soils

The extra-green method was adopted to segment crop and weed from soil background. The color index of extra-green was computed as the formula:

Extra-green $=2 \mathrm{G}-\mathrm{R}-\mathrm{B}$

2) Detection of between-row weed based on position feature

The pixel histogram of green plant was used to determine the center line and the width of crop row, and then distinguish the between-row weed.

3) Discrimination of intra-row weed based on texture feature 
The block of texture was selected on the basis of the center line of crop row. The co-occurrence matrixes of the $\mathrm{H}$ color space were computed. Based on it, five texture parameters were extracted. Then, the K-means clustering method was used to recognized weed within crop rows.

The method of crop and weed segmentation line used the $\mathrm{H}$ and $\mathrm{S}$ color space and the distance clustering method. The nonlinear model of segmentation crop and weed was built by the statistic data. The correct classification rate of weed cluster was achieved $90 \%$, and the mistake classification rate of crop was lowed to $5 \%$.

\section{ULTRA-LOW ALTITUDE REMOTE SYSTEM FOR CAPTURING FIELD INFORMATION}

The study of ultra-low altitude remote system provided a good hardware platform for field information gathering in precision agriculture (Figure 6). Its establishment prepared for observation of plant diseases and insect pests in field, on measurement of the crop growing and field data, etc.

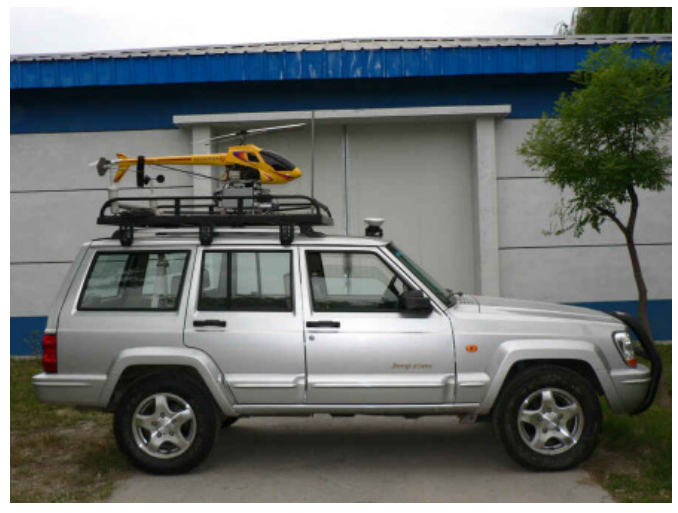

Figure 6. An ultra-low altitude remote system

The technical indicators of the ultra-low altitude remote system were controllable flight height $(2-200 \mathrm{~m})$, maximum speed $(120 \mathrm{Km} / \mathrm{h})$ and load ability $(>4 \mathrm{~kg}$ ). The helicopter loaded $5.7 \mathrm{~kg}$ could work in order. The test result showed that the system could be dynamic flight indeed the condition of two meters or under.

For capturing field information, the ultra-low altitude flight system mainly mounted visible light detectors in the visible light. Hot infrared, near infrared and Doppler's detectors could also be loaded for special survey. The automatic detectors is equipped with wireless data communication system of high speed (1Mbps), allocating large capacity data memory $(>40 \mathrm{G})$, and data 
automatic analysis and process system for remote information. The regional GIS and expert system of insect pest and weeds were set up. Weeds and insect distribution message were display with the graphical method.

\section{CONCLUSION}

The novel research on precision agricultural equipment has been accomplished in the near years. The industrialization research on precision agricultural technical equipment and products will be developed to turn the precision agricultural technology into production.

\section{REFERENCES}

Liu Jiyu. Principle and method on GPS satellite navigation and position. Beijing: Science Press, 2003:229-261.

Zhang Xiaochao, Wang Yiming, Wang Youxiang. Application and study on GPS technology for irrigation system. Transactions of the Chinese society for agricultural machinery, 2004, 35(6):102-105. 